\title{
Textural radiomic features and time- intensity curve data analysis by dynamic contrast-enhanced MRI for early prediction of breast cancer therapy response: preliminary data
}

\author{
Roberta Fusco ${ }^{1 *}$, Vincenza Granata ${ }^{1}$, Francesca Maio ${ }^{2}$, Mario Sansone ${ }^{3}$ and Antonella Petrillo ${ }^{1}$
}

\begin{abstract}
Background: To investigate the potential of semiquantitative time-intensity curve parameters compared to textural radiomic features on arterial phase images by dynamic contrast-enhanced magnetic resonance imaging (DCE-MRI) for early prediction of breast cancer neoadjuvant therapy response.

Methods: A retrospective study of 45 patients subjected to DCE-MRI by public datasets containing examination performed prior to the start of treatment and after the treatment first cycle ('QIN Breast DCE-MRI' and 'QIN-Breast') was performed. In total, 11 semiquantitative parameters and 50 texture features were extracted. Non-parametric test, receiver operating characteristic analysis with area under the curve (ROC-AUC), Spearman correlation coefficient, and Kruskal-Wallis test with Bonferroni correction were applied.

Results: Fifteen patients with pathological complete response $(p C R)$ and 30 patients with non-pCR were analysed. Significant differences in median values between $p C R$ patients and non-pCR patients were found for entropy, long-run emphasis, and busyness among the textural features, for maximum signal difference, washout slope, washin slope, and standardised index of shape among the dynamic semiquantitative parameters. The standardised index of shape had the best results with a ROC-AUC of 0.93 to differentiate pCR versus non-pCR patients.
\end{abstract}

Conclusions: The standardised index of shape could become a clinical tool to differentiate, in the early stages of treatment, responding to non-responding patients.

Keywords: Breast neoplasms, Magnetic resonance imaging, Neoadjuvant therapy, Radiomics

\section{Key points}

- Significant differences between pathological complete response (pCR) and non-pCR patients were found for texture parameters.

- Standardised Index of shape (SIS) showed the highest accuracy to differentiate pCR patients from non-pCR patients.

\footnotetext{
* Correspondence: r.fusco@istitutotumori.na.it

${ }^{1}$ Radiology Division, Istituto Nazionale Tumori - IRCCS - Fondazione G.

Pascale, Via Mariano Semmola, Naples, Italy

Full list of author information is available at the end of the article
}

- SIS could become a clinical tool to differentiate early responders by non-responders.

\begin{abstract}
Background
Breast cancer is the most common cancer diagnosed in the USA [1]. Neoadjuvant therapy (NAT) has been recommended in locally advanced disease $[2,3]$ to determine a downstaging for a following resection to increase tumour control likelihood and breast-conserving surgery rate [4]. Pathologic complete response (pCR) after NAT has been found to be related with long-term clinical benefit, such as disease-free and overall survival $[5,6]$.
\end{abstract}


Dynamic contrast-enhanced magnetic resonance imaging (DCE-MRI), being a non-invasive imaging method to measure tissue microvascular perfusion and permeability, is used in clinical trials and research settings to assess NAT response [7]. In clinical settings, changes in tumour size are usually used to assess breast cancer response to NAT. However, changes in tumour size often were found to manifest later compared with changes in vascular tumour functions [8]. There is extensive literature showing that semiquantitative [9] or quantitative pharmacokinetic analysis [10] of DCE-MRI data can provide better prediction, also in early phase, of breast cancer pathologic response to NAT than tumour size changes.

Previous studies have investigated functional parameters derived from DCE-MRI to assess neoadjuvant treatment such as the standardised index of shape (SIS) proposed by Petrillo et al. [11-15] as a simple semiquantitative feature capable to predict pathological significant response and pathological complete response (pCR) after chemo-radiation therapy or after short course radiotherapy. Petrillo et al. demonstrated the ability of SIS to predict $\mathrm{pRC}$ and pathological significant response after preoperative chemo-radiotherapy in locally advanced rectal cancer [11-15]. Moreover, texture analysis from breast DCE-MRI has been shown to be effective in applications such as automatic lesion segmentation $[16,17]$ and cancer diagnosis $[18,19]$.

Here, we conducted a radiomic analysis of statistical texture features extracted by arterial phase of DCE-MRI and semiquantitative dynamic parameters for early prediction of breast cancer response to NAT. We report our preliminary findings on the performance of these two kinds of data.

\section{Methods}

\section{Dataset characteristics}

Two public dataset were used: 'QIN Breast DCE-MRI' and 'QIN-Breast'.

The public dataset 'QIN Breast DCE-MRI' from The Cancer Imaging Archive (TCIA) collection [20, 21] is composed of ten patients subjected to DCE-MRI using a Siemens 3-T TIM Trio system with the body coil and a four-channel bilateral phased-array breast coil. Axial bilateral DCE-MRI images with fat saturation and full breast coverage were acquired with a three-dimensional gradient echo-based time-resolved angiography with stochastic trajectories sequence. DCE-MRI acquisition parameters included echo time $2.9 \mathrm{~ms}$ and repetition time $6.2 \mathrm{~ms}$; field of view $30-34 \mathrm{~cm}$, in-plane matrix size $320 \times 320$; and slice thickness $1.4 \mathrm{~mm}$. The total acquisition time was about $10 \mathrm{~min}$ for 32-34 image volume sets of 112-120 slices each, with a temporal resolution of 18-20 s. The contrast agent was Gd-HP-DO3A, gadoteridol (Bracco Imaging, Milan, Italy), intravenously injected $(0.1 \mathrm{mmol} / \mathrm{kg}$ at $2 \mathrm{~mL} / \mathrm{s})$ by a programmable power injector timed to commence after acquisition of two baseline image volumes, followed by a $20-\mathrm{mL}$ saline flush. The public data set can be downloaded at https:// wiki.cancerimagingarchive.net/display/Public/QIN+ Breast+DCE-MRI.

The public dataset 'QIN-Breast' from The Cancer Imaging Archive (TCIA) collection [21, 22] is composed of 35 patients subjected to DCE-MRI using a 3-T Philips Achieva system using a dedicated 16-channel bilateral breast coil. Axial bilateral DCE-MRI images with fat saturation and full breast coverage were acquired with a radiofrequency spoiled three-dimensional gradient echo sequence. Acquisition parameters included echo time 7.9 and repetition time $4.6 \mathrm{~ms}$, field of view $22 \mathrm{~cm}^{2}$, inplane matrix size $192 \times 192$, and slice thickness $5 \mathrm{~mm}$. For the DCE study, each 20-slice set was collected in 16 $\mathrm{s}$ at 25 time points for just under $7 \mathrm{~min}$ of dynamic scanning. The contrast agent was Gd-DTPA and gadopentetate dimeglumine (Bayer Health Care Pharmaceuticals, Wayne, NJ, USA) was intravenously injected (0.1 $\mathrm{mmol} / \mathrm{kg}$ at $2 \mathrm{~mL} / \mathrm{s}$ ) by a programmable power injector timed to commence after acquisition of two baseline image volumes, followed by a $20-\mathrm{mL}$ saline flush. The public data set can be downloaded at https://wiki.cancer imagingarchive.net/display/Public/QIN-Breast.

The NAT protocol administered to these patients was left to the discretion of the treating oncologist based on patient factors such as menopausal status and age as well as tumour characteristics, including size, grade, nodal status and receptor status and was reported by $\mathrm{Li}$ et al. in [22]. Both these collections of breast DCE-MRI data contain images from two studies to assess NAT response. Images were acquired at two time points: before and after the first cycle of treatment.

\section{Data analysis}

Manual segmentation was performed by an expert breast radiologist (with a 25-year experience) on the postcontrast arterial phase images, drawing manually each slice to obtain the delineating of the whole tumour contours (volume of interest).

\section{Textural features}

We considered 50 textural features, including both firstorder features (mean, mode, median, standard deviation [SD], median absolute deviation, range (absolute difference between maximum and minimum values), kurtosis, skewness, and interquartile range) and second-order features. Calculations were performed using the 'TextureToolbox' of MATLAB R2007a (MathWorks, Natick, MA, USA) that performs texture analysis from an input by region or volume of interest. In particular, this 
texture analysis package allows for wavelet band-pass filtering, isotropic resampling, discretisation length corrections and different quantitation tools. A detailed description has been provided by Vallières et al. [23]. The toolbox can be downloaded at https:/it.mathworks.com/ matlabcentral/fileexchange/51948-radiomics. The definition of significant textural features reported in the "Results" section is provided in Additional file 1.

\section{Semiquantitative dynamic parameters}

A time-intensity curve can be subdivided into three regions. The first one represents the contrast medium time needed to reach the lesion, and the signal intensity is equal to the basal level before contrast agent injection; the second one shows the increase in signal intensity because of contrast medium absorption (washin) according to the tumour biology; the third one mainly represents the backflow of the contrast medium into the plasma (washout). To estimate shape descriptors, a piecewise linear fitting was made and ten semiquantitative dynamic features described in the literature [24-26] were extracted using the approach reported in a previous publication from our group [25], maximum signal difference (MSD), time to peak between washin and washout segments, washin slope (WIS), washout slope (WOS), washin intercept, washout intercept, area under the curve of washin, area under the curve of washout, and area under the curve of washin and washout. The last semiquantitative dynamic feature was the SIS obtained combining linearly the percentage change of MSD and WOS. Therefore, for SIS calculation, the percentage change of MSD $[\triangle \mathrm{MSD}=(\mathrm{MSD} 1-\mathrm{MSD} 2) /$ MSD1 $\times 100]$, and of WOS $[\triangle \mathrm{WOS}=(\mathrm{WOS} 1$ WOS2)/WOS1 $\times 100]$ and their combination as previously described [11] was evaluated. Standardised SIS was given by the following linear combination: $0.7780 * \Delta$ MSD $+0.6157 * \Delta$ WOS. In order to evaluate the SIS, an OsiriX (Pixmeo SARL, Geneva, Switzerland) plugin has been developed by the authors.

\section{Reference standard and pathological methods}

The reference standard was the pathology from surgical specimen. Fifteen pCR patients and 30 non-pCR patients were included in this retrospective study. The pCR was classified according to Miller-Payne grade: grade 1 for no reduction, grade 2 for minor loss ( $\leq$ $30 \%$ ), grade 3 for loss from 30 to $90 \%$, grade 4 for marked loss (>90\%), and grade 5 for no residual invasive cancer. Patients with grades $1,2,3$, or 4 were scored as non-pCR.

\section{Statistical analysis}

Median, SD, and range were calculated as representative values of segmented volumes of interest. Percentage change of median values of parameters obtained before and after the first cycle of treatment was calculated. Receiver operating characteristic analysis was used for obtaining the area under the curve (ROC-AUC). Sensitivity, specificity, positive predictive value (PPV), negative predictive value (NPV), and accuracy were obtained considering the optimal cutoff values identified maximising the Youden index.

For two-group comparisons, we used the nonparametric Kruskal-Wallis test for continuous variables. A $p$ value $<0.05$ was considered as significant for univariate analysis. Bonferroni correction was applied for multiple comparisons.

Calculations were performed using the Statistics and Machine Learning Toolbox of MATLAB R2007a (MathWorks, Natick, USA).

\section{Results}

Table 1 reports the median, SD, and range of the percentage change for the significant features in the differentiation $\mathrm{pCR}$ from non-pCR patients. Significant differences in median values between $\mathrm{pCR}$ patients and non-pCR patients using the Kruskal-Wallis test were found for entropy, long-run emphasis (LRE), and busyness among the textural features and for MSD, WOS, WIS and SIS among the dynamic parameters.

Table 2 reports accuracy for the significant features: entropy (accuracy 71\%), LRE (accuracy 71\%), busyness (accuracy 76\%), MSD (accuracy 78\%), WIS (accuracy 78\%), WOS (accuracy 82\%), and SIS (accuracy 89\%). The SIS showed the best performance with a ROC-AUC of 0.93, a sensitivity of $93 \%$, a specificity of $87 \%$, a PPV of $78 \%$, and a NPV of the $96 \%$, using an optimal cutoff value of $56.47 \%$ to differentiate pCR from non-pCR patients. The SIS increased the accuracy of $13 \%$ respect to the better parameter among texture features, of $11 \%$ compared to MSD and WIS and of $7 \%$ respect to WOS.

In Fig. 1, boxplots for the significant textural features (entropy, LRE, busyness) and dynamic features (WIS, WOS, MSD, and SIS) to separate pCR from non-pCR patients are reported. Moreover, Fig. 1 shows ROC-AUC curves for all significant parameters (entropy, LRE, busyness, WIS, WOS, MSD, SIS). In Fig. 2, a case of nonpCR is shown: morphological images did not show a significant change in tumour size while there was a significant modification in time-intensity curve before and after the first cycle of treatment while the SIS value was $72.3 \%$.

\section{Discussion}

Recent advances in biomedical image analysis have emphasised that MRI contrast kinetic parameters and 
Table 1 Median, standard deviation and range of the percentage change for significant features differentiating patients with pathologic complete response ( $\mathrm{pCR}$ ) from non-pCR patients

\begin{tabular}{llrrrrrrr}
\hline & & $\Delta$ Entropy (\%) & $\Delta$ LRE (\%) & $\Delta$ Busyness (\%) & $\Delta$ MSD (\%) & $\Delta$ WIS [\%] & $\Delta$ WOS [\%] & SIS (\%) \\
\hline Non-pCR patients & Median & 0.28 & 0.29 & 23.79 & 12.49 & 38.52 & -11.43 & 9.59 \\
& SD & 7.30 & 2.28 & 75.93 & 38.94 & 50.21 & 95.11 & 63.80 \\
& Range & 30.57 & 12.60 & 298.18 & 234.22 & 210.05 & 420.02 & 320.65 \\
pCR patients & Median & 5.03 & 1.30 & 38.83 & 68.89 & 87.35 & 143.67 & 125.17 \\
& SD & 11.69 & 1.84 & 37.75 & 45.30 & 47.37 & 129.24 & 251.58 \\
& Range & 43.82 & 7.52 & 134.56 & 142.17 & 155.66 & 492.36 & 779.98 \\
\multirow{3}{*}{ Total } & Median & 2.16 & 0.52 & 27.67 & 17.00 & 50.54 & 20.74 & 27.96 \\
& SD & 9.57 & 2.21 & 68.69 & 43.92 & 51.28 & 130.15 & 183.57 \\
& Range & 43.82 & 12.60 & 307.92 & 250.40 & 224.91 & 641.45 & 993.42 \\
& & 0.021 & 0.024 & 0.023 & 0.014 & 0.012 & $<0.001$ & $<0.001$ \\
\hline
\end{tabular}

Range represents the absolute difference between maximum and minimum values

LRE Long-run emphasis, MSD Maximum signal difference, SD Standard deviation, SIS Standardised index of shape, WIS Washin slope, WOS Washout slope *Kruskal-Wallis test

texture analysis, as quantitative metrics, can offer a refined local tumour description of complexity, heterogeneity and kinetic behaviour [27-29].

Teruel et al. [30] presented the findings on 16 textural statistical features extracted by DCE-MRI that are capable to predict early NAT tumour response. Golden et al. [27] used similar texture features to predict $\mathrm{pCR}$, residual lymph node metastases and residual tumour in patients with triplenegative breast cancer. Moreover, Thibault et al. [28] reported that breast tumour microvasculature heterogeneity as a texture feature could be a useful biomarker for early prediction of NAT response. However, these studies used statistical texture description without taking advantage of information provided by the T1-weighted DCE-MRI curve.

Martincich et al. [29] showed that a reduction in the tumour volume $>65 \%$ and a reduction in the early enhancement ratio after two cycles of preoperative therapy were associated with a major histopathological response. Combining tumour volume and early enhancement ratio reduction after two cycles of therapy reached a 93\% diagnostic accuracy to identify $\mathrm{pCR}$.

We have extracted multiple statistical texture features on arterial phase of DCE-MRI and semi-quantitative kinetic parameters before and after one cycle of NAT in order to assess early pathological response using two public dataset acquired with 3T MR scanner. Our monovariate analysis shows statistically positive results for entropy (71\% of accuracy), LRE (71\% of accuracy), busyness among texture features (76\% of accuracy) and for MSD (78\% of accuracy), WIS (78\% of accuracy), WOS ( $82 \%$ of accuracy), and SIS (89\% of accuracy) among semi-quantitative kinetic metrics. Textural feature results for entropy, LRE and busyness confirmed the results presented by Thibault et al. [28], suggesting changes in the spatial heterogeneity of the tumour microenvironment as one of the initial NAT effects.

Moreover, our perfusion and permeability as semiquantitative dynamic parameters, measured by contrast

Table 2 Diagnostic accuracy for significant features differentiating patients with pathologic complete response ( $p C R$ ) versus non-pCR patients

\begin{tabular}{|c|c|c|c|c|c|c|c|c|c|}
\hline & & $p$ value ${ }^{*}$ & ROC-AUC & Sensitivity & Specificity & PPV & NPV & Accuracy & Cutoff \\
\hline \multirow[t]{3}{*}{ Textural features } & $\Delta$ Entropy & 0.024 & 0.71 & 0.67 & 0.73 & 0.56 & 0.81 & 0.71 & 3.78 \\
\hline & $\Delta \mathrm{LRE}$ & 0.021 & 0.71 & 0.73 & 0.70 & 0.55 & 0.84 & 0.71 & 0.57 \\
\hline & $\Delta$ Busyness & 0.020 & 0.72 & 0.67 & 0.80 & 0.63 & 0.83 & 0.76 & 34.38 \\
\hline \multirow[t]{4}{*}{ Dynamic features } & $\Delta \mathrm{MSD}$ & 0.013 & 0.74 & 0.67 & 0.83 & 0.67 & 0.83 & 0.78 & 27.74 \\
\hline & $\Delta \mathrm{WIS}$ & $<0.001$ & 0.73 & 0.60 & 0.87 & 0.69 & 0.81 & 0.78 & 73.62 \\
\hline & $\triangle$ WOS & 0.012 & 0.86 & 0.87 & 0.80 & 0.68 & 0.92 & 0.82 & 24.42 \\
\hline & SIS & $<0.001$ & 0.93 & 0.93 & 0.87 & 0.78 & 0.96 & 0.89 & 56.47 \\
\hline
\end{tabular}

LRE Long-run emphasis, MSD Maximum signal difference, NPV Negative predictive value, PPV Positive predictive value, ROC-AUC Receiver operating characteristic area under the curve, SIS Standardised index of shape, WIS Washin, WOS Washout slope *Kruskal-Wallis test 

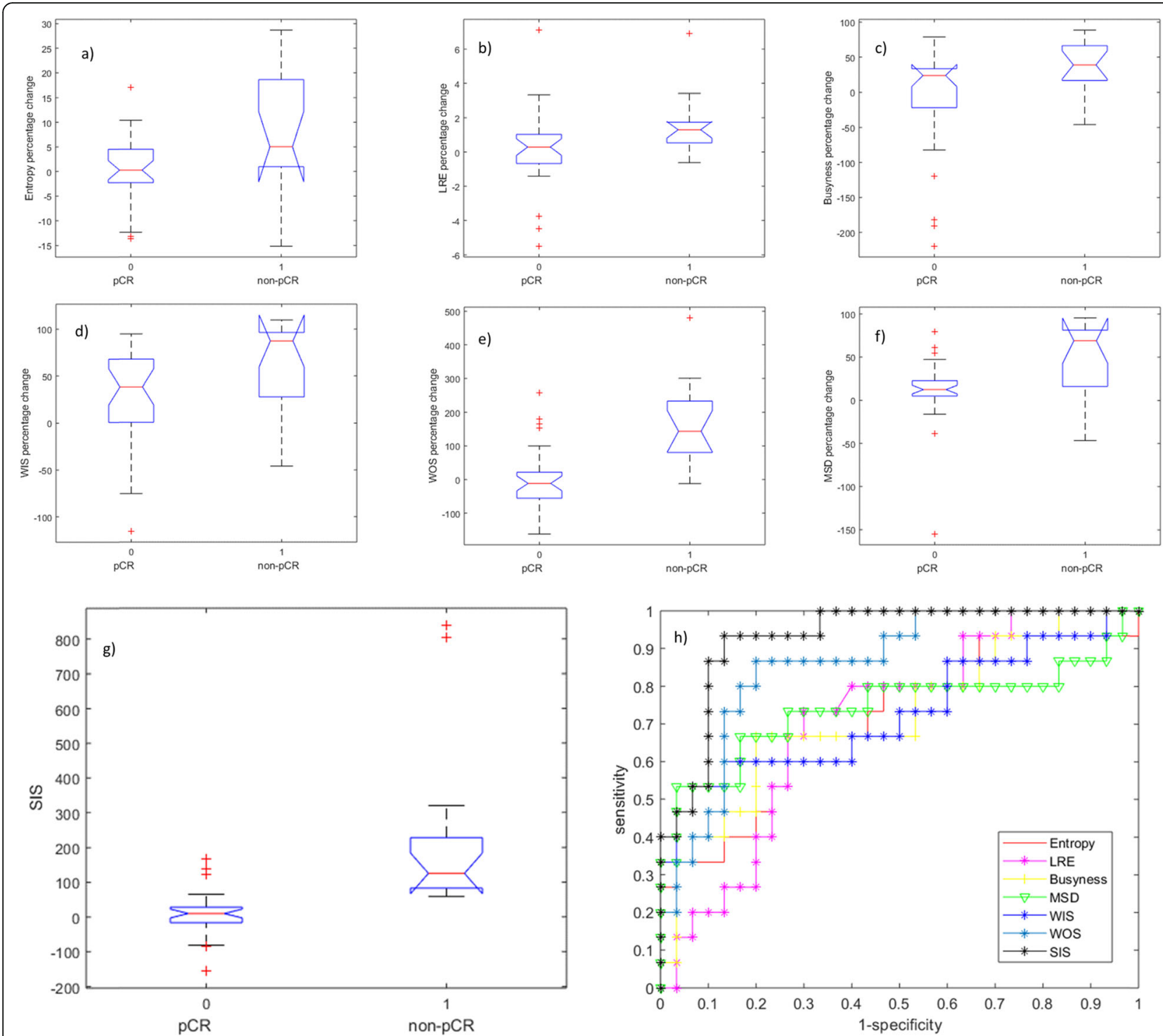

Fig. 1 Boxplots for those metrics significantly separating patients with pathologic complete response (pCR) from non-pCR patients. Textural features: (a) entropy, (b) long-run emphasis (LRE) and (c) busyness. Dynamic parameters: (d) washin slope (WIS), (e) washout slope (WOS), (f) maximum signal difference (MSD), (g) standardised index of shape (SIS). $\mathbf{h}$ Receiver operating characteristic area under the curve for all these significant metrics

kinetics, have reported good results, especially WOS and SIS, indicating that changes in DCE-MRI are important markers for identifying early pCR [11, 24, 29].

However, SIS analysis reached the best results in terms of sensitivity, specificity, PPV, and NPV, reporting the highest ROC-AUC value (0.93) for predicting pCR. With the optimal cutoff value, SIS increases the accuracy of $13 \%$ compared to the better parameter among texture features, of $11 \%$ compared to MSD and WIS and of $7 \%$ compared to WOS.

This study has several limitations. First of all, this pool of patients derives from two different public datasets created in two different hospitals with two different MR machines using two different sequence tools. Second, the small cohort of studied patients represents an initial finding to validate increasing sample size of the study in the future. Third, NAT regimen is not available for each patient because the analysed MR images were obtained by public dataset. Finally, this analysis did not consider tumour histological differences. In fact, the potential integration of texture, morphological and dynamic metrics combined with histopathology results may provide other important prognostic information for the assessment and the prediction of therapy response. 


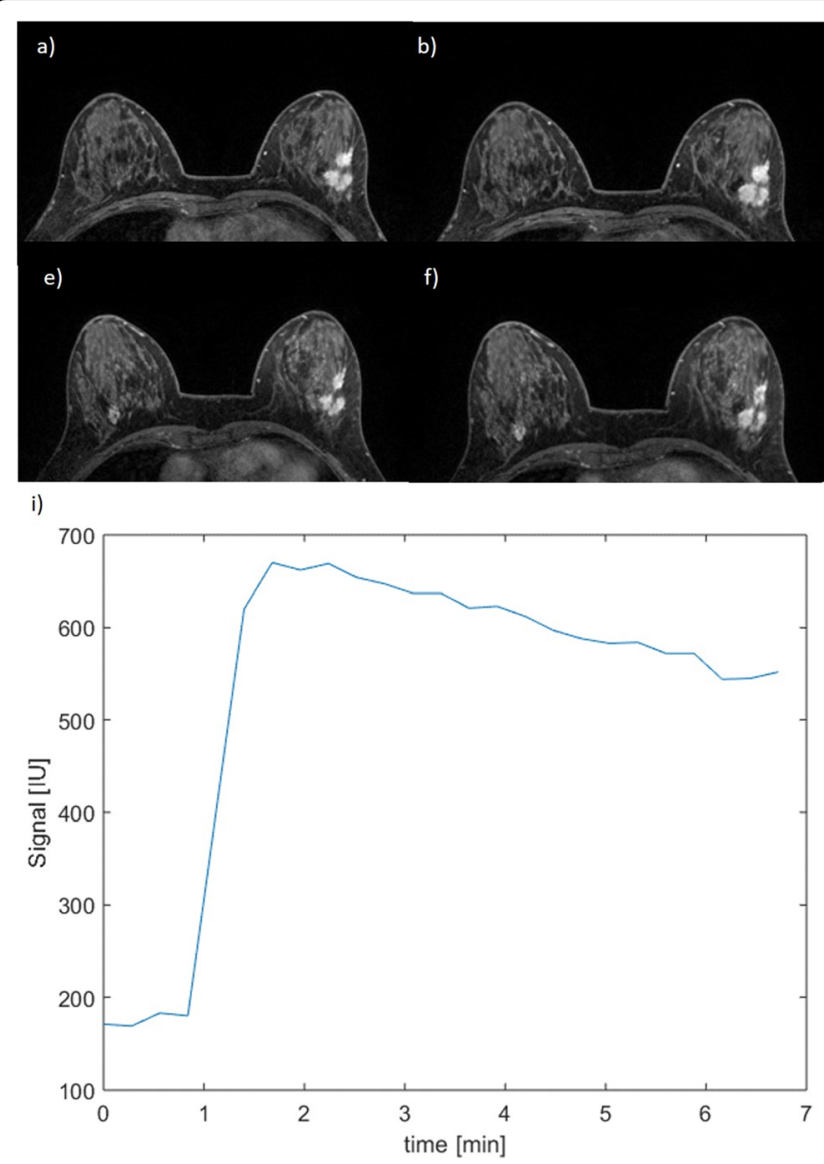

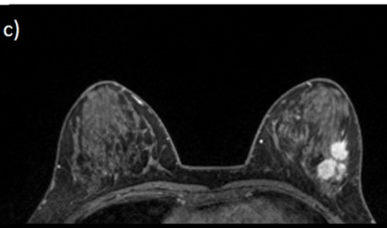

g) d)

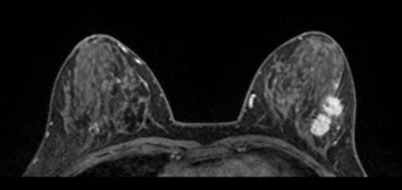

h)

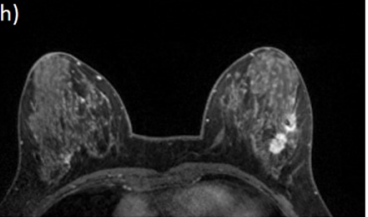

j)

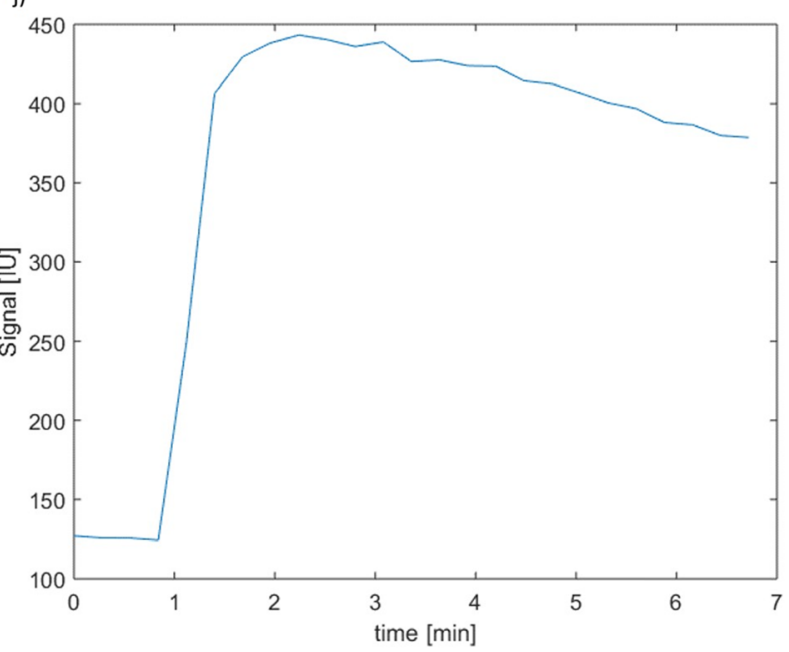

Fig. 2 A case of a patient with non-pathologic complete response. a-d Four slices of T1-weighted dynamic contrast-enhanced magnetic resonance imaging before treatment. $\mathbf{e}-\mathbf{h}$ Four slices of $\mathrm{T} 1$-weighted dynamic contrast-enhanced magnetic resonance imaging after the first cycle of treatment. i Pre-treatment time-intensity curve and (j) time-intensity curve after the first cycle of treatment

In conclusion, although validation in larger patient populations is needed, feature extraction approach and SIS can become important clinical tools to identify and differentiate, in the early stages of NAT treatment, responding and non-responding patients for alternative personalised therapy regimens.

\section{Supplementary information}

Supplementary information accompanies this paper at https://doi.org/10. 1186/s41747-019-0141-2.

Additional file 1. Definition of significant texture features

\section{Abbreviations}

DCE-MRI: Dynamic contrast-enhanced magnetic resonance imaging; LRE: Long-run emphasis; MSD: Maximum signal difference; NAT: Neoadjuvant therapy; NPV: Negative predictive value; pCR: Pathological complete response; PPV: Positive predictive value; ROC-AUC: Receiver operating characteristic area under the curve; SIS: Standardised index of shape; WIS: Washin slope; WOS: Washout slope

\section{Acknowledgements}

The authors are grateful to Alessandra Trocino, librarian at the National Cancer Institute of Naples, Italy.

\section{Authors' contributions}

AP conceived of the study and participated in its design and coordination and in drafting the manuscript. RF, MS, and FM participated in the study collection, analysed the data and drafted the manuscript. AP and VG participated in the study collection. All authors read and approved the final manuscript.

Funding

The authors declare that this work has not received any funding

Availability of data and materials

The datasets used and/or analysed during the current study are available from the corresponding author on reasonable request.

Ethics approval and consent to participate

Not applicable.

\section{Consent for publication}

Not applicable.

\section{Competing interests}

The authors declare that they have no competing interests.

\section{Author details}

${ }^{1}$ Radiology Division, Istituto Nazionale Tumori - IRCCS - Fondazione G. Pascale, Via Mariano Semmola, Naples, Italy. ${ }^{2}$ Radiology Division, Universita' Degli Stui di Napoli Federico II, Via Pansini, Naples, Italy. ${ }^{3}$ Department of 
Electrical Engineering and Information Technologies (DIETI), University of Naples Federico II, Via Claudio, Naples, Italy.

Received: 17 September 2019 Accepted: 5 December 2019 Published online: 05 February 2020

\section{References}

1. DeSantis CE, Ma J, Goding Sauer A, Newman LA, Jemal A (2017) Breast cancer statistics, 2017, racial disparity in mortality by state. CA Cancer J Clin 67:439-448. https://doi.org/10.3322/caac.21412

2. Peart O (2015) Breast intervention and breast cancer treatment options. Radiol Technol 86:535 M-55558

3. Kaufmann M, von Minckwitz G, Mamounas EP et al (2012) Recommendations from an international consensus conference on the current status and future of neoadjuvant systemic therapy in primary breast cancer. Ann Surg Oncol 19:1508-1516. https://doi.org/10.1245/ s10434-011-2108-2

4. Rubovszky G, Horváth Z (2017) Recent advances in the neoadjuvant treatment of breast cancer. J Breast Cancer 20:119-131. https://doi.org/10. 4048/jbc.2017.20.2.119

5. De Los Santos J, Bernreuter W, Keene K et al (2011) Accuracy of breast magnetic resonance imaging in predicting pathologic response in patients treated with neoadjuvant chemotherapy. Clin Breast Cancer 11:312-319. https://doi.org/10.1016/j.clbc.2011.06.007

6. Bonnefoi H, Litière S, Piccart M et al (2014) Pathological complete response after neoadjuvant chemotherapy is an independent predictive factor irrespective of simplified breast cancer intrinsic subtypes: a landmark and two-step approach analyses from the EORTC 10994/BIG 1-00 phase III trial. Ann Oncol 25:1128-1136. https://doi.org/10.1093/annonc/mdu1 18

7. Marinovich ML, Sardanelli F, Ciatto S et al (2012) Early prediction of pathologic response to neoadjuvant herapy in breast cancer: systematic review of the accuracy of MRI. Breast 21:669-677. https://doi.org/10.1016/j. breast.2012.07.006

8. O'Connor JP, Jackson A, Parker GJ, Roberts C, Jayson GC (2012) Dynamic contrast-enhanced MRI in clinical trials of antivascular therapies. Nat Rev Clin Oncol 9:167-177. https://doi.org/10.1038/nrclinonc.2012.2

9. Pickles MD, Lowry M, Manton DJ, Turnbull LW (2015) Prognostic value of DCE-MRI in breast cancer patients undergoing neoadjuvant chemotherapy: a comparison with traditional survival indicators. Eur Radiol 25:1097-1106. https://doi.org/10.1007/s00330-014-3502-5

10. Tudorica A, Oh KY, Chui SY et al (2016) Early prediction and evaluation of breast cancer response to neoadjuvant chemotherapy using quantitative DCE-MRI. Transl Oncol 9:8-17. https://doi.org/10.1016/j.tranon.2015.11.016

11. Petrillo A, Fusco R, Petrillo M et al (2015) Standardized index of shape (SIS): a quantitative DCE-MRI parameter to discriminate responders by nonresponders after neoadjuvant therapy in LARC. Eur Radiol 25:1935-1945. https://doi.org/10.1007/s00330-014-3581-3

12. Petrillo A, Fusco R, Granata V et al (2018) Assessing response to neoadjuvant therapy in locally advanced rectal cancer using intra-voxel incoherent motion modelling by DWI data and Standardized Index of Shape from DCE-MRI. Ther Adv Med Oncol 16;10:1758835918809875. doi: https://doi.org/10.1177/1758835918809875.

13. Fusco R, Sansone M, Granata V et al (2019) Diffusion and perfusion MR parameters to assess preoperative short-course radiotherapy response in locally advanced rectal cancer: a comparative explorative study among standardized index of shape by DCE-MRI, intravoxel incoherent motion- and diffusion kurtosis imaging-derived parameters. Abdom Radiol (NY). 44:36833700. https://doi.org/10.1007/s00261-018-1801-z

14. Petrillo A, Fusco R, Granata V et al (2017) MR imaging perfusion and diffusion analysis to assess preoperative short course radiotherapy response in locally advanced rectal cancer: standardized index of shape by DCE-MRI and intravoxel incoherent motion-derived parameters by DW-MRI. Med Oncol 34:198. https://doi.org/10.1007/s12032-017-1059-2

15. Petrillo A, Fusco R, Petrillo M et al (2017) Standardized index of shape (DCEMRI) and standardized uptake value (PET/CT): two quantitative approaches to discriminate chemo-radiotherapy locally advanced rectal cancer responders under a functional profile. Oncotarget 8:8143-8153. https://doi. org/10.18632/oncotarget.14106

16. Szabó BK, Aspelin P, Wiberg MK (2004) Neural network approach to the segmentation and classification of dynamic magnetic resonance images of the breast: comparison with empiric and quantitative kinetic parameters. Acad Radiol 11:1344-1354. https://doi.org/10.1016/j.acra.2004.09.006

17. Woods BJ, Clymer BD, Kurc T et al (2007) Malignant-lesion segmentation using $4 \mathrm{D}$ co-occurrence texture analysis applied to dynamic contrastenhanced magnetic resonance breast image data. J Magn Reson Imaging 25:495-501. https://doi.org/10.1002/jmri.20837

18. Agner SC, Rosen MA, Englander $S$ et al (2014) Computerized image analysis for identifying triple-negative breast cancers and differentiating them from other molecular subtypes of breast cancer on dynamic contrast-enhanced MR images: a feasibility study. Radiology 272:91-99. https://doi.org/10.1148/ radiol.14121031

19. Chaudhury B, Zhou M, Goldgof DB et al (2015) Heterogeneity in intratumoral regions with rapid gadolinium washout correlates with estrogen receptor status and nodal metastasis. J Magn Reson Imaging 42: 1421-1430. https://doi.org/10.1002/jmri.24921

20. Huang W, Li X, Chen Y, Li X et al (2014) Variations of dynamic contrastenhanced magnetic resonance imaging in evaluation of breast cancer therapy response: a multicenter data analysis challenge. Trans Oncol 7:153166. https://doi.org/10.1593/tlo.13838

21. Clark K, Vendt B, Smith K et al (2013) The cancer imaging archive (TCIA): maintaining and operating a public information repository. J Digit Imaging 26:1045-1057. https://doi.org/10.1007/s10278-013-9622-7

22. Li X, Abramson RG, Arlinghaus LR et al (2015) Multiparametric magnetic resonance imaging for predicting pathological response after the first cycle of neoadjuvant chemotherapy in breast cancer. Invest Radiol 50:195-204. https://doi.org/10.1097/RLL.0000000000000100

23. Vallières M, Freeman CR, Skamene SR, El Naqa I (2015) A radiomics model from joint FDG-PET and MRI texture features for the prediction of lung metastases in soft-tissue sarcomas of the extremities. Phys Med Biol 60: 5471-5496. https://doi.org/10.1088/0031-9155/60/14/5471

24. Fusco R, Di Marzo M, Sansone C, Sansone M, Petrillo A (2017) Breast DCEMRI: lesion classification using dynamic and morphological features by means of a multiple classifier system. Eur Radiol Exp1 10. https://doi.org/10. 1186/s41747-017-0007-4

25. Fusco R, Petrillo A, Petrillo M, Sansone M (2013) Use of tracer kinetic models for selection of semi-quantitative features for DCE-MRI data classification. Applied Magnetic Resonance 44:1311-1324. https://doi. org/10.1007/s00723-013-0481-7

26. Fusco R, Sansone M, Filice $S$ et al (2015) Integration of DCE-MRI and DWMRI quantitative parameters for breast lesion classification. Biomed Res Int 2015:237863. https://doi.org/10.1155/2015/237863

27. Golden DI, Lipson JA, Telli ML, Ford JM, Rubin DL (2013) Dynamic contrastenhanced MRI-based biomarkers of therapeutic response in triple-negative breast cancer. J Am Med Inform Assoc 20:1059-1066. https://doi.org/10. 1136/amiajnl-2012-001460

28. Thibault G, Tudorica A, Afzal A et al (2017) DCE-MRI texture features for early prediction of breast cancer therapy response. Tomography 3:23. https://doi.org/10.18383/.jom.2018.00046

29. Martincich L, Montemurro F, De Rosa G et al (2004) Monitoring response to primary chemotherapy in breast cancer using dynamic contrast-enhanced magnetic resonance imaging. Breast Cancer Res Treat 83:67-76. https://doi. org/10.1023/B:BREA.0000010700.11092.f4

30. Teruel JR, Heldahl MG, Goa PE et al (2014) Dynamic contrast-enhanced MRI texture analysis for pretreatment prediction of clinical and pathological response to neoadjuvant chemotherapy in patients with locally advanced breast cancer. NMR Biomed 27:887-896. https://doi.org/10.1002/nbm.3132

\section{Publisher's Note}

Springer Nature remains neutral with regard to jurisdictional claims in published maps and institutional affiliations. 